\title{
Correlation between EGFR Gene Mutations and Lung Cancer: a Hospital-Based Study
}

\author{
Kavitha Matam ${ }^{1}$, Iravathy Goud ${ }^{1}$, Adi Maha Lakshmi M1ㅗ Ravi $\mathbf{V}^{1}$, Sridhar \\ $K^{1}$, Vijayanand Reddy P $^{1}$, Srinivas Chakravarthy ${ }^{2}$, SVSS Prasad ${ }^{2}$, Shaik Nazia \\ Tabassum $^{3}$, Noor Ahmad Shaik ${ }^{4}$, Rabbani Syed ${ }^{5}$, Khalid Khalaf Alharbi ${ }^{5,6,7}$, \\ Imran Ali Khan ${ }^{5 *}$
}

\begin{abstract}
Epidermal growth factor receptor $(E G F R)$ is one of the targeted molecular markers in many cancers including lung malignancies. Gefitinib and erlotinib are two available therapeutics that act as specific inhibitors of tyrosine kinase (TK) domains. We performed a case-control study with formalin-fixed paraffin-embedded tissue blocks (FFPE) from tissue biopsies of 167 non-small cell lung carcinoma (NSCLC) patients and 167 healthy controls. The tissue biopsies were studied for mutations in exons 18-21 of the EGFR gene. This study was performed using PCR followed by DNA sequencing. We identified 63 mutations in 33 men and 30 women. Mutations were detected in exon 19 (delE746-A750, delE746-T751, delL747-E749, delL747-P753, delL747-T751) in 32 patients, exon $20(\mathrm{S786I}, \mathrm{T790M})$ in 16 , and exon $21(\mathrm{L858R})$ in 15. No mutations were observed in exon 18 . The 63 patients with EFGR mutations were considered for upfront therapy with oral tyrosine kinase inhibitor (TKI) drugs and have responded well to therapy over the last 15 months. The control patients had no mutations in any of the exons studied. The advent of $E G F R$ TKI therapy has provided a powerful new treatment modality for patients diagnosed with NSCLC. The study emphasizes the frequency of EGFR mutations in NSCLC patients and its role as an important predictive marker for response to oral TKI in the south Indian population.
\end{abstract}

Keywords: $E F G R$ - NSCLC - TKI - DNA sequencing

Asian Pac J Cancer Prev, 16 (16), 7071-7076

\section{Introduction}

Lung Cancer is the most common cancer and the leading cause of cancer death in the world. This is also true in India, where cancer accounts for $28 \%$ of the total number of deaths. The incidence of lung cancer in Asia has become the greatest threat to human health (Varalakshmi et al., 2013; Zhao et al., 2014). The etiological factors affecting lung cancer have not been completely uncovered, but some studies propose that environmental exposure to tobacco smoke is a primary risk factor (He et al., 2014). Other known carcinogens related to lung cancer are radon, arsenic, cadmium, chromates, and asbestos (Wang et al., 2014). Lung cancer is divided into non-small-cell lung cancer (NSCLC) and small-cell lung cancer. Adenocarcinoma, squamous cell carcinoma, and small-cell carcinoma are three major forms of lung cancers. Therefore, the pivotal role of genetics in cancer predisposition, especially in the most malignant lung cancers, has prompted substantial interest in recent years (Jin et al., 2014).

Mutations in the epidermal growth factor receptor $(E G F R)$ gene are reported far more frequently in lung cancer than any other cancer (Powrozek et al., 2014). $E G F R$ mutation analysis is one of the best predictive markers for the use of EGFR-Tyrosine Kinase Inhibitor (TKI) therapy in NSCLC (Thunnissen et al., 2014). EGFR mutations may also impart an improved overall prognosis for advanced NSCLC patients compared with $E G F R$ wild-type tåumors. The predictive and prognostic role of EGFR mutational status in stage I through III NSCLC remains less well defined, with conflicting results in studies reported to date. In many of these studies, the administration of neoadjuvant or adjuvant chemotherapy or TKIs clouds the interpretation of the impact of EGFR status on prognosis. The EGFR gene shows significant

${ }^{1}$ Molecular Biology and Cytogenetics Department, Apollo Health city Building, Apollo Hospitals, ${ }^{2}$ Department of Oncology, Apollo Hospitals, Jubilee Hills, Hyderabad, ${ }^{3}$ Department of Pharmacy practice, Browns college of Pharmacy, Khammam, Telangana, India, ${ }^{4}$ Department of Genetic Medicine, Faculty of Medicine, King Abdulaziz University, Jeddah, ${ }^{5}$ Department of Clinical Laboratory Sciences, ${ }^{6}$ Saudi society for Clinical Laboratory Sciences, ${ }^{7}$ Medical and Molecular Genetics Research Chair, College of Applied Medical Sciences, King Saud University, Riyadh, Kingdom of Saudi Arabia*For correspondence: imkhan@ksu.edu.sa 
and durable response to treatment with the EGFR-TKI erlotinib or gefitinib (Izar et al., 2013). Single-nucleotide polymorphisms (SNPs) are the most common and stable markers of human genetic variation, and may be associated with the risk of a variety of cancers, including that of the lung (He et al., 2014). Deletions in exon 19 and the L858R point mutation in exon 21 occur most frequently and are associated with a response rate of approximately $70 \%$ to EGFR-TKI therapy (Thunnissen et al., 2014).

We performed this study to examine the prognostic value of EGFR mutation status in NSCLC patients in a cosmopolitan city in the South of India. We aimed to identify the impact of EGFR mutational status on the risk of lung cancer, and the NSCLC patients' clinical outcome when treated with oral EGFR-TKI. We carried out a hospital-based study in the former capital city of Hyderabad, India. We investigated the hypothesis that EGFR mutations may be an important indicator of lung cancer and may be correlated with clinical characteristics.

\section{Materials and Methods}

\section{Selection of subjects}

This is a prospective case-control hospital-based study carried out in the Department of Molecular Biology and Ctytogenetics at the Apollo Hospital, in Telangana region of Hyderabad, India. Histological type was determined according to WHO criteria. In this study, 274 subjects were included and among them 167 patients were randomly selected from the Oncology department. This study included only patients with NSCLC, confirmed by postoperative pathology, who were receiving EGFRTKI treatment. Normal healthy controls $(n=167)$ were selected from the general population and included in our study. Freshly harvested NSCLC biopsies were fixed in $10 \%$ buffered formalin within $30 \mathrm{~min}$ and embedded into paraffin (FFPE). Immunohistochemistry (IHC) staining was performed on two cores extracted from each specimen. Written informed-consent was obtained for each patient and the ethical committee of the Apollo Hospital approved the study.

\section{DNA isolation and genotyping of EGFR mutations}

Genomic DNA was isolated from FFPE lung biopsies of NSCLC patients and control subjects as per the standard QIAamp protocol recommended by the manufacturers (QIAGEN Inc., Valencia, CA). The quality and quantity of the DNA was quantified using a spectrophotometer. The purity was determined by calculating the ratio of absorbance at $260 \mathrm{~nm}$ to absorbance at $280 \mathrm{~nm}$ (A260/ A280). Non-annealed DNA should have an A260/A280 ratio of 1.7-1.9. Polymerase chain reaction (PCR) was performed in a thermal cycler for exons 18, 19, 20, and 21 with flanking intronic sequences. Specific primers were designed for the selected exons in the EGFR gene. The details of the selected exons and primer sequences are listed in Table 1. The PCR profile consisted of 35 cycles of denaturation $\left(95^{\circ} \mathrm{C}\right.$ for $\left.5 \mathrm{~min}\right)$, annealing (at $52^{\circ} \mathrm{C}$ for $30 \mathrm{~s}$ ), and extension at $\left(72^{\circ} \mathrm{C}\right.$ for $5 \mathrm{~min}$ ) (Khan et al., 2015). The amplified products were separated on $2 \%$ agarose gel, stained with ethidium bromide (Cambrex,
East Rutherford, NJ, USA), and visualized on a UV transilluminator (Dafco, USA). DNA Sanger sequencing method were used (Khan et al., 2015a).

\section{Sanger sequencing}

The purified PCR fragments were then sequenced in both the forward and reverse directions. Chain termination sequencing involves the synthesis of new strands of DNA complementary to a single-stranded template. The template DNA ( $\sim 50 \mathrm{ng})$ was purified with a PCR clean up kit and was amplified in a total reaction volume of $20 \mu \mathrm{L}$ containing Big Dye terminator reaction buffer, forward and reverse primers (10 pmoles each), and molecular grade water. DNA sequencing was performed with an Applied Biosystem machine. The reaction was performed at $95^{\circ} \mathrm{C}$ for $15 \mathrm{~s}, 60^{\circ} \mathrm{C}$ for $15 \mathrm{~s}$, and $60^{\circ} \mathrm{C}$ for $5 \mathrm{~min}$. The amplified gene in the reaction was precipitated after several washes in $95 \%$ and $70 \%$ alcohol, dried in a vacuum centrifuge, resuspended in Hi-Di formamide, and loaded onto 7200 Genetic Analyzer (Applied Biosystem, Chicago, USA) for sequencing. The resultant sequences were compared with the Cambridge sequence and Seascapes software.

\section{Statistical analysis}

Clinical characteristics of all subjects are expressed as the mean \pm SD. Alleles and genotype frequency differences between patients and controls were tested using a chisquare test. Odds ratios (ORs) and 95\% confidence intervals were calculated by binomial logistic regression for the allele, genotype, and haplotype frequencies, and the chi-square test was used to identify departures from Hardy-Weinberg equilibrium. Statistical analyses were performed with SPSS (version 19.0) software.

\section{Results}

\section{Baseline characteristics}

167 cases and 167 controls were included in this study. All subjects were native of Hyderabad, India. In the study case subjects, $112(67 \%)$ were male and 55 $(33 \%)$ were female. The control subjects consisted of $110(65.9 \%)$ males and 57 (34.1\%) females. The baseline characteristics are shown in Table 2. The mean age was 55.12 years in cases and 51.12 in controls $(\mathrm{p}=0.56)$. In this study, $90(53.9 \%)$ subjects were smokers and the remaining $77(46.1 \%)$ were non-smokers. The patients who were selected for this study had NSCLC tumors $(n=167)$, whereas the control subjects all had benign tumors $(n=50)$.

\section{Mutational analysis}

Genotyping was performed with direct sequencing in all 167 patients and 167 controls. Sixty-three (37.7\%) patients had an EGFR mutation in all exons tested (1821), as determined by Sanger sequencing. All the NSCLC patients had at least a single mutation and none of the patients had a double mutation (i.e. not more than 1 mutation in Exon 18-21). The most frequently observed EGFR mutation was a deletion (Del 19) on the LREA region of exon 19 , found in $32(19.1 \%)$ patients. The second most common EGFR mutation, T790M on exon 
20, was found in $16(9.6 \%)$ of the NSCLC patients. T790M has been implicated in primary and secondary resistance to EGFR-TKIs. The exon 21 point mutation L858R was found in 15 (9\%) patients (Table 3). No mutations were observed on exon 18 (G719). Genotyping and sequencing was also performed in the control subjects $(n=167)$, however we did not find any mutations in any of the tested exons (exon18-21) (Figures 1 and 2).

\section{Gender stratification}

We compared the stratification of mutations in the selected exons based on gender and found $29.2 \%$ of the mutations were male-specific, while $54.5 \%$ of the mutations were female-specific. A total of $18.7 \%(n=26)$ mutations were found in males on exon $19,3.5 \%(n=4)$ were in exon 20 , and $7.1 \%(n=4)$ were in exon 21 . In females we found $20 \%(\mathrm{n}=11)$ of the total mutations in exon $19,21.8 \%(n=12)$ in exon 20 , and $12.7 \%(n=7)$ in exon 21 . There were no mutations observed in exon 18 (Table 4).

Table 1. Exon Regions Amplified, Primers Sequences Used in the Study

\begin{tabular}{|c|c|c|}
\hline $\begin{array}{c}\text { S. } \\
\text { No }\end{array}$ & Exon & Primer sequences \\
\hline \multirow{2}{*}{1} & \multirow{2}{*}{$\begin{array}{c}\text { Exon } \\
18\end{array}$} & F: 5'-AGG GCT GAG GTG ACC CTT GT-3' \\
\hline & & R: 3'-TCC CCA CCA GAC CAT GAG AG -5 \\
\hline \multirow[t]{2}{*}{2} & \multirow{2}{*}{$\begin{array}{c}\text { Exon } \\
19\end{array}$} & $\begin{array}{c}\text { F:5'-ACC ATC TCA CAA TTG CCA GTT } \\
\text { AAC - } 3^{\prime}\end{array}$ \\
\hline & & R:3'-GAG GTT CAG AGC CAT GGA CC -5' \\
\hline \multirow{2}{*}{3} & \multirow{2}{*}{$\begin{array}{c}\text { Exon } \\
20\end{array}$} & $\begin{array}{c}\text { F:5'-GAT CGC ATT CAT GCG TCT TCA CC } \\
-3^{\prime}\end{array}$ \\
\hline & & $\begin{array}{c}\text { R:3'-TTG CTA TCC CAG GAG CGC AGA } \\
\text { CC }-5^{\prime}\end{array}$ \\
\hline \multirow[t]{2}{*}{4} & \multirow{2}{*}{$\begin{array}{c}\text { Exon } \\
21\end{array}$} & $\begin{array}{c}\text { F:5'-TCA CAG CAG GGT CTT CTC TGT } \\
\text { TT - } 3^{\prime} \\
\end{array}$ \\
\hline & & R:3'-ATG CTG GCT GAC CTA AAG CC -5' \\
\hline
\end{tabular}

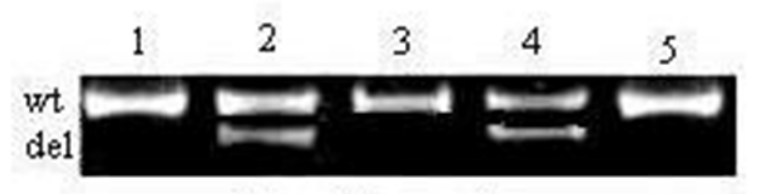

Exon 19 mutation

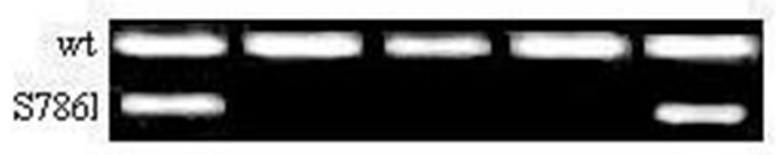

Exon 20 mutation

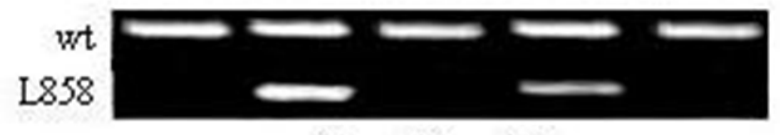

Exon 21 mutation

Figure 1. Gel Images of Exon 19, 20 and 21

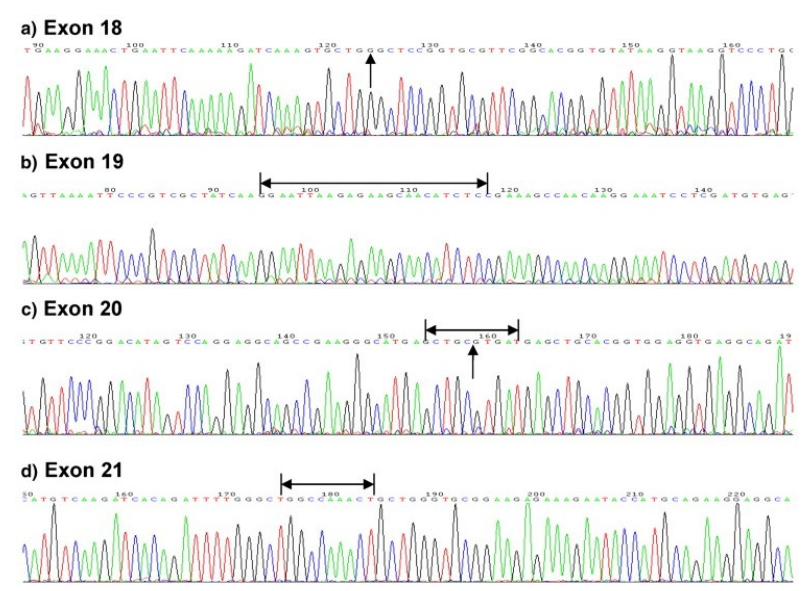

Figure 2. The Chromatogram of EGFR Mutational Analysis for Exons 18-21. The arrows indicate expected location of missense, deletion and insertion mutations

Table 2. Patient demographics, clinical characteristics and details of EGFR mutation

\begin{tabular}{lccc}
\hline Characteristics & Patients $(\mathrm{n}=167)$ & Controls $(\mathrm{n}=167)$ & $\mathrm{p}$ Value \\
\hline Age (Years) & $55.14 \pm 10.22$ & $51.12 \pm 9.77$ & 0.56 \\
Gender (M/F) & $112(67 \%) / 55(33 \%)$ & $110(65.9 \%) / 57(34.1 \%)$ & 0.64 \\
Smokers & $90(53.9 \%)$ & $87(52.1 \%)$ & 0.66 \\
Non-Smokers & $77(46.1 \%)$ & $80(47.1 \%)$ & 0.78 \\
Adenocarcinoma & - & - & NA \\
Squamous cell carcinoma & - & - & NA \\
Adenosquamous carcinoma & - & - & NA \\
NSCLC & $167(100 \%)$ & - & NA \\
Control Benign & - & $167(100 \%)$ & NA \\
Stage & $21(12.6 \%)$ & - & NA \\
IIIb & $126(75.4 \%)$ & - & NA \\
IV & $20(12 \%)$ & - & NA \\
Postoperative relapse & & - & NA \\
Performance Status & $164(98.2 \%)$ & - & NA \\
$0-1$ & $02(1.2 \%)$ & - & NA \\
2 & $01(0.6 \%)$ & & \\
$4-$ Mar & & & \\
\hline
\end{tabular}

N/A= Not applicable 
Table 3. Mutation Detection in the Cases and Controls of the EGFR Gene

\begin{tabular}{lcc}
\hline Exon & Cases $(\mathrm{n}=167)$ & controls $(\mathrm{n}=167)$ \\
\hline Exon 18 & $0(0)$ & $0(0)$ \\
Exon 19 & $32(19.1 \%)$ & $0(0)$ \\
Exon 20 & $16(9.6 \%)$ & $0(0)$ \\
Exon 21 & $15(9 \%)$ & $0(0)$ \\
\hline
\end{tabular}

Table 4. Effects of Gender Status on EGFR Mutation

\begin{tabular}{lcc}
\hline Exon & Males $(\mathrm{n}=112)$ & Females $(\mathrm{n}=55)$ \\
\hline Exon 18 & $0(0)$ & $0(0)$ \\
Exon 19 & $21(18.7)$ & $11(20)$ \\
Exon 20 & $4(3.57)$ & $12(21.81)$ \\
Exon 21 & $8(7.14)$ & $7(12.72)$ \\
\hline
\end{tabular}

Table 5. Effects of Smoking on EGFR Mutations

\begin{tabular}{lcc}
\hline Exon & Smokers $(\mathrm{n}=90)$ & Non-smokers $(\mathrm{n}=77)$ \\
\hline Exon 18 & $0(0)$ & $0(0)$ \\
Exon 19 & $26(28.9 \%)$ & $10(13 \%)$ \\
Exon 20 & $15(16.7 \%)$ & $2(2.6 \%)$ \\
Exon 21 & $9(10 \%)$ & $1(1.3 \%)$ \\
\hline
\end{tabular}

\section{Smoking}

Furthermore, we analyzed our study with respect to patient smoking status. Our calculations indicated that $55.5 \%(\mathrm{n}=50)$ of the total mutations were present in the tumor biopsies isolated from smokers, while $16.9 \%$ $(n=13)$ of the mutations were observed in the non-smokers' tumor biopsies. In smokers, we found $28.9 \%(\mathrm{n}=26)$ of the mutations in exon $19,16.7 \%(\mathrm{n}=15)$ in exon 20 , and $10 \%(n=9)$ of the total mutations in exon 21 . However, in non-smokers we found $13 \%(\mathrm{n}=10)$ of the total mutations in exon $19,2.6 \%(n=2)$ in exon 20 , and $1.3 \%(n=1)$ in exon 21. There were no mutations in exon 18 (Table 5).

\section{Discussion}

We scrutinized the influence of $E G F R$ mutations on lung cancer risk by conducting a hospital-based casecontrol study. EGFR is one of the most highly targeted molecular markers in many cancers including lung cancer. Genetic modifications such as deletions, insertions, and SNP in the TK domain of EGFR are a common feature observed in most lung cancers. We studied the prevalence of EGFR mutations in NSCLC patients from samples obtained from biopsy/cytology/pleural fluid and fine needle aspiration (FNA), across South India. We have screened for 13 somatic mutations that span exons 18, 19, 20, and 21 of EGFR gene using PCR and Sanger sequencing. The underlying reason for the high somatic mutation rate noted in this study is uncertain. All of the cases included in the current study had been confirmed to have NSCLC tumors, as reported in the majority of the literature. This also may reflect the finding that cases in the current study were more selective because at the study institution a specific molecular test was often requested by clinicians in consultation with pathologists after reviewing the clinicopathologic characteristics of each individual case (Cai et al., 2013).

Gefitinib is an oral EGFR TKI that has been shown to be efficacious and well tolerated in patients with pretreated advanced NSCLC (Kris et al., 2003; Fukuoka et al., 2013). The complex relationship between $E G F R$ related biomarkers and response to $E G F R$-TKIs has been investigated extensively. EGFR mutation testing was recommended prior to systemic chemotherapy for all patients with advanced NSCLC, excluding SCC (David et al., 2006). To select the appropriate treatment regimen, rapid and accurate mutation test results are necessary in clinical practice. Although various methods are used to detect $E G F R$ mutations, there is no universal consensus on which method is the most effective. However, studies have shown that direct DNA sequencing and TaqManbased real-time PCR followed by pyrosequencing are the current standard for EGFR mutation detection (Ettinger et al., 2010).

Previous studies have suggested that $-216 \mathrm{G} / \mathrm{T}$ (Reinersman et al., 2010; Noronha et al., 2013) and D994D (Paez et al., 2004) polymorphisms are associated with clinical outcome of gefitinib therapy. CA-SSR in intron 1 of EGFR is the most studied polymorphism. CA-SSR has been associated with EGFR gene expression and has been reported to correlate with clinical outcome of gefitinib therapy (Reinersman et al., 2010; Sun and Ueno et al., 2012; Noronha et al., 2013). Shorter CA repeats have been associated with higher transcription levels of $E G F R$ and have been reported to be correlated with better clinical outcome of gefitinib therapy. Liu et al found that the $-216 \mathrm{G} / \mathrm{T}$ polymorphism and CA-19 genotypes are found more frequently in patients with exon 19 deletions (Liu et al., 2011). On the other hand, Suzuki et al reported that the EGFR protein expression level was significantly higher in the shorter CA repeats group than in the longer allele group, but its length was not associated with $E G F R$ somatic mutations (Suzuki et al., 2008). Jou et al. (2009) revealed that the EGFR $8227 \mathrm{G} / \mathrm{A}$ polymorphism was associated with lung cancer, especially in non smoking female lung adenocarcinoma patients in the Taiwanese population (Jou et al., 2009; Shitara et al., 2012).

In our study, $37.7 \%$ of the patients were found to harbor an EGFR mutation. The previous study from India found that the mutation rate was 35\% (24), which was similar to our study. It is likely that the present study and the prior Indian report overestimated the incidence of EGFR mutations because of small sample sizes and clinically selected patients. Worldwide, the incidence of EGFR mutations has been well characterized and has been reported to occur at a rate of 10-15\% in North Americans and Europeans, 19\% in African-Americans, and about $30 \%$ in East Asians (Paez et al., 2004; Cortes-Funes et al., 2005; Reinersman et al., 2010; Dong et al., 2012).

In this study, we found that 13 (16.9\%) of patients with EGFR mutations were non-smokers, while 50 (55.5\%) of the patients who did not have any EGFR mutations had a smoking history (Table 5). Our study also indicated that smokers have more frequent $E G F R$ mutations as compared to non-smokers (Table 5). This is in contrast to the studies by Sun et al. (2012) and Noronha et al (2013), which showed that EGFR mutations were more 
frequent in females than in males and in non-smokers than smokers. The reason for these inconsistencies in our study is currently unknown; however, it may be due to our small sample size and the greater number of male smokers included in our study group.

In our study, the highest frequency of mutations was seen in exon 19, followed by exon 20 , and exon 21 (Table 3). Similar observations were made by Chang et al who found more $E G F R$ mutations in patients who are Asian, female, nonsmokers, and have adenocarcinoma (Kosaka et al., 2004; Marchetti et al., 2005; Shigematsu et al., 2005; Tokumo et al., 2005; Chang et al., 2006).

In a similar study by Shigematsu et al. (2005) and Rosell et al. (2009), the most common EGFR mutations were short, in-frame deletions (most often 15 or $18 \mathrm{bp}$ ) in exon 19. Our study group did not show any mutations in exon 18. A similar observation was made by Noronha et al (2013) where $74 \%$ of patients were noted to have an in-frame deletion in exon 19, while $23 \%$ had the L858R point mutation in exon 21 , and only $2.5 \%$ of patients had the $\mathrm{G} 719 \mathrm{C}$ point mutation in exon 18 . There was a positive association between the number of EGFR mutations and age amongst never-smokers regardless of sex, indicating that EGFR mutations occur cumulatively by unidentified internal/external factors other than smoking. Aging is one of the best, but rarely referred, risk factors for various types of cancer including lung cancer, because age could be a surrogate for accumulation of genetic events in cancers. Smoking is inversely associated with the presence of EGFR mutations in lung cancer, but because smoking status is strongly confounded by age and sex, the sole impact of age is difficult to evaluate. Our patients with EGFR-activating mutations had a significantly better response rate, progression-free survival, and overall survival when treated with EGFR-targeted therapies. This result was seen similarly by Noronha et al. (2013).

In this study, $80 \%$ of the positive EGFR patients were treated with an oral TKI. Among the 36 patients, only 24 patients $(66.6 \%)$ responded to the oral TKI therapy and survived for 15 months (Table 6). The remaining 12 patients harboring activating mutations in the EGFR tyrosine kinase domain were found to be resistant to oral TKI at their 2- to 3-month follow-up scan, and survived for 9 months. This might be possibly due to exon 20 mutations, which are considered to be TK resistant (Wang et al., 2013). Thus, it is necessary to identify more markers for the effective prediction of patient response to $E G F R$ TKIs, and it is also necessary to obtain biopsies of the primary tumor subsequently during the course of treatment to detect the presence of secondary mutations that could alter the patient's drug response (Pao et al., 2004). Thus, Indian patients with EGFR-activating mutations have a significantly better response rate and progression-free survival when treated with EGFR targeted therapies.

To summarize, the advent of EGFR TKI therapy has provided a powerful new treatment modality for patients diagnosed with NSCLC. Yet, primary and acquired resistance to targeted therapy continues to be a major obstacle for satisfying clinical outcomes. Thus, the identification of specific molecular alterations that contribute to $E G F R$-targeted therapy response has become critical for selecting patients for appropriate treatments. We found that among the mutant positive cases, the deletions delE746-A750 in exon 19 and a missense mutation L858 in exon 21 were the most predominant. Therefore, these prognostic indicators, and can be used as biomarkers to customize treatment for a particular patient.

\section{Acknowledgements}

We are thankful to all the volunteers who had participated in this study. We are grateful to the Apollo hospitals for supporting this study.

\section{References}

Cai G, Wong R, Chhieng D, et al (2013). Identification of EGFR mutation, KRAS mutation, and ALK gene rearrangement in cytological specimens of primary and metastatic lung adenocarcinoma. Cancer Cytopathol, 121, 500-7.

Chang A, Parikh P, Thongprasert S, et al (2006). Gefitinib (IRESSA). in patients of Asian origin with refractory advanced non-small cell lung cancer: subset analysis from the ISEL study. $J$ Thorac Oncol, 1, 847-55.

Cortes-Funes H, Gomez C, Rosell R, et al (2005). Epidermal growth factor receptor activating mutations in Spanish gefitinib-treated non-small-cell lung cancer patients. Ann Oncol, 16, 1081-6.

David M Jackman, Beow Y Yeap, Lecia V Sequist Neal Lindeman, et al (2006). Exon 19 deletion mutations of epidermal growth factor receptor are associated with prolonged survival in non-small cell lung cancer patients treated with gefitinib or erlotinib. Clin Cancer Res, 12, 3908.

Dong J, Hu Z, Wu C, et al (2012). Association analyses identify multiple new lung cancer susceptibility loci and their interactions with smoking in the Chinese population. Nat Genet, 44, 895-9.

Ettinger DS, Akerley W, Bepler G, et al (2010). Non-small cell lung cancer. J Natl Compr Canc Netw, 8, 740-801.

He J, Deng L, Na F, et al (2014). The association between TGF- $\beta 1$ polymorphisms and radiation pneumonia in lung cancer patients treated with definitive radiotherapy: a metaanalysis. PLoS One, 9, 91100.

He P, Yang XX, He XQ, et al (2014). CHRNA3 polymorphism modifies lung adenocarcinoma risk in the Chinese han population. Int J Mol Sci, 15, 5446-57.

Izar B, Sequist L, Lee M, et al (2013). The impact of EGFR mutation status on outcomes in patients with resected stage I non-small cell lung cancers. Ann Thorac Surg, 96, 962-8.

Jin F, Qian C, Qing Y, et al (2014). Genetic polymorphism of APE1 rs1130409 can contribute to the risk of lung cancer. Tumour Biol, 35, 6665-71.

Jou YS, Lo YL, Hsiao CF, et al (2009). Association of an $E G F R$ intron $1 \mathrm{SNP}$ with never smoking female lung adenocarcinoma patients. Lung Cancer, 64, 251-6.

Kosaka T, Yatabe Y, Endoh H, et al (2004). Mutations of the epidermal growth factor receptor gene in lung cancer: biological and clinical implications. Cancer Res, 64, 89198923.

Liu W, He L, Ramirez J, et al (2011). Functional EGFR germ line polymorphisms may confer risk for $E G F R$ somatic mutations in non-small cell lung cancer, with a predominant effect on exon 19 microdeletions. Cancer Res, 71, 2423-7.

Fukuoka M, Yano S, Giaccone G, et al (2003). Multi-institutional randomized phase II trial of gefitinib for previously treated patients with advanced non-small-cell lung cancer (the IDEAL trial). J Clin Oncol, 21, 2237-46. 


\section{Kavitha Matam et al}

Khan IA, Shaik NA, Kamineni V, et al (2015). Evaluation of gestational diabetes mellitus risk in south indian women based on MTHFR (C677T) and FVL (G1691A) mutations. Front Pediatr, 34, 1-5.

Khan IA, Shaik NA, Pasupuleti N, et al (2015a). Screening of mitochondrial mutations and insertion-deletion polymorphism in gestational diabetes mellitus in the Asian Indian population. Saudi J Bio Sci, 22, 243-8.

Kris MG, Natale RB, Herbst RS, et al (2003). Efficacy of gefitinib, an inhibitor of the epidermal growth factor receptor tyrosine kinase, in symptomatic patients with non-small cell lung cancer: a randomized trial. J Am Med Assoc, 290, 2149-58.

Marchetti A, Martella C, Felicioni L, et al (2005). EGFR mutations in non- small-cell lung cancer: analysis of a large series of cases and development of a rapid and sensitive method for diagnostic screening with potential implications on pharmacologic treatment. J Clin Oncol, 23, 857-65.

Noronha V, Prabhash K, Thavamani A, et al (2013). EGFR mutations in Indian lung cancer patients: clinical correlation and outcome to EGFR targeted therapy. PLOS ONE, 8, 61561.

Paez JG, Janne PA, Lee JC, et al (2004). EGFR mutations in lung cancer: correlation with clinical response to gefitinib therapy. Science, 304, 1497-500.

Pao W, Miller V, Zakowski M, et al (2004). EGF receptor gene mutations are common in lung cancers from "never smokers" and are associated with sensitivity of tumors to gefitinib and erlotinib. Proc Natl Acad Sci USA, 101, 13306-11.

Powrozek T, Krawczyk P, Ramlau R, et al (2014). EGFR gene mutations in patients with adenosquamous lung carcinoma. Asia Pac J Clin Oncol, 10, 340-5.

Reinersman JM, Johnson ML, Riely GJ, et al (2010). Frequency of $E G F R$ and KRAS mutations in lung adenocarcinomas in African Americans. J Thorac Oncol, 6, 28-31.

Rosell R, Moran T, Queralt C, et al (2009). Screening for epidermal growth factor receptor mutations in lung cancer. N Engl J Med, 361, 958-67.

Sahoo R, Harini VV, Babu VC, et al (2011). Screening for EGFR mutations in lung cancer, a report from India. Lung Cancer, 73, 316-9.

Shigematsu H, Lin L, Takahashi T, et al (2005). Clinical and biological features associated with epidermal growth factor receptor gene mutations in lung cancers. J Natl Cancer Inst, 97, 339-46.

Shitara M, Sasaki H, Yokota K, et al (2012). Polymorphisms in intron 1 of the EGFR gene in non-small cell lung cancer patients. Exp Ther Med, 4, 785-9.

Sun PL, Seol H, Lee HJ, et al (2012). High incidence of EGFR mutations in Korean men smokers with no intratumoral heterogeneity of lung adenocarcinomas: correlation with histologic subtypes, EGFR/TTF-1 expressions, and clinical features. J Thorac Oncol, 7, 323-30.

Suzuki M, Kageyama S, Shinmura K, et al (2008). Inverse relationship between the length of the EGFR CA repeat polymorphism in lung carcinoma and protein expression of EGFR in the carcinoma. J Surg Oncol, 98, 457-461.

Thunnissen E, van der Oord K, den Bakker M (2014). Prognostic and predictive biomarkers in lung cancer. A review. Virchows Arch, 464, 347-58.

Tokumo M, Toyooka S, Kiura K, et al (2005). The relationship between epidermal growth factor receptor mutations and clinicopathologic features in non-small cell lung cancers. Clin Cancer Res, 11, 1167-73.

Ueno T, Toyooka S, Suda K, Soh J, Yatabe Y, Miyoshi S, Matsuo K, Mitsudomi T. et al (2012). Impact of age on epidermal growth factor receptor mutation in lung cancer. Lung Cancer,
78, 207-11.

Varalakshmi K (2013). Classification of lung cancer nodules using a hybrid approach. J Emerg Trends Comput Informat Sci, 4, 63-68.

Wang B, Zhang L, Qiu F, et al (2014). A newfound association between mdc1 functional polymorphism and lung cancer risk in Chinese. PLoS One, 9, 106794.

Wang Y, Wenlong B, Hua S, et al (2013). Epidermal growth factor receptor exon 20 mutation increased in post-chemotherapy patients with non-small cell lung cancer detected with patients' blood samples. Transl Oncol, 4, 504-10.

Zhao Y, Wei Q, Hu L, et al (2014). Polymorphisms in microRNAs are associated with survival in non-small cell lung cancer. Cancer Epidemiol Biomarkers Prev, 23, 2503-11. 